\title{
TWO NOTES ON CONVERGENCE AND DIVERGENCE A.E. OF FOURIER SERIES WITH RESPECT TO SOME ORTHOGONAL SYSTEMS
}

\author{
J. J. GUADALUPE, M. PEREZ, F. J. RUIZ, AND J. L. VARONA
}

(Communicated by J. Marshall Ash)

\begin{abstract}
We study some problems related to convergence and divergence a.e. for Fourier series in systems $\left\{\phi_{k}\right\}$, where $\left\{\phi_{k}\right\}$ is either a system of orthonormal polynomials with respect to a measure $d \mu$ on $[-1,1]$ or a Bessel system on $[0,1]$. We obtain boundedness in weighted $L^{p}$ spaces for the maximal operators associated to Fourier-Jacobi and Fourier-Bessel series. On the other hand, we find general results about divergence a.e. of the Fourier series associated to Bessel systems and systems of orthonormal polynomials on $[-1,1]$.
\end{abstract}

\section{INTRODUCTION}

Let $d u$ be a positive measure on a finite interval $[a, b] \subset \mathbb{R}$ and

$$
S_{n} f(x)=\sum_{k=1}^{n} c_{k} \phi_{k}(x), \quad c_{k}=\int_{a}^{b} f(t) \phi_{k}(t) d \mu(t),
$$

the $n$th partial sum of the Fourier series of $f$ with respect to a real, complete, and orthonormal system $\left\{\phi_{k}\right\}$ on $L^{2}(d \mu)$.

In what follows $\left\{\phi_{k}\right\}$ will be either the system of orthonormal polynomials given by the normalization of the sequence $\left\{1, x, x^{2}, \ldots\right\}$ in $L^{2}([-1,1] ; d \mu)$ or the Bessel system in $L^{2}([0,1] ; d \mu)$, where $d \mu=x d x$.

We shall denote by $S^{*} f(x)=\sup _{n}\left|S_{n} f(x)\right|$ the maximal operator associated to the Fourier series of $f$. We are interested in the two following questions:

(a) Given $p \in[1,2)$, does $S_{n} f(x)$ converge to $f(x) \mu$ a.e. for any $f$ in $L^{p}(d \mu)$ ?

(b) Is $S^{*}$ of strong or weak type $(p, p)$ ?

Very well-known standard arguments show that an affirmative answer to question (b) leads to an affirmative answer to question (a). Several authors have studied the former questions in the case of orthonormal polynomials on $[-1,1]$ or Bessel systems on [0,1]. Titchmarsh [16] and Benedek-Panzone [3] analyze

Received by the editors January 8, 1990 and, in revised form, March 18, 1991.

1991 Mathematics Subject Classification. Primary $42 \mathrm{C} 10$.

Key words and phrases. Fourier series, orthonormal polynomials, maximal operators, $A_{p}$ weights.

Research supported by CAICYT PB85-0338 and DGICYT PB89-0181-C02-02. 
question (a) in the case of Fourier-Bessel series, determinating the range of $p$ 's such that (a) is true, but their techniques (equiconvergence theorems) cannot be used to approach matter (b).

For Jacobi polynomials, equiconvergence theorems again show that there exists a range of $p$ 's such that (a) is true (see [15]). Badkov studies in [2] the problem of the boundedness of the operator $S^{*}$ for generalized Jacobi polynomials. He obtains boundedness (even in some weighted $L^{p}$-spaces) for the same range of $p$ 's in which (a) is true.

With respect to negative answers of question (a), Pollard [14] shows that for each $p<\frac{4}{3}$ there exists a function $f \in L^{p}(d x)$ such that its Legendre-Fourier series diverges a.e. After that, Meaney [10] extends the result to $p=\frac{4}{3}$ and to Jacobi polynomials (see also Badkov [2]). In these results, the asymptotic behavior of the polynomials is used together with category arguments.

Our purpose in this paper is two fold. First we obtain results about boundedness of the maximal operators in weighted $L^{p}$ spaces, which extend Badkov's results in the case of Jacobi polynomials and are new in the case of Bessel functions (even in the unweighted case). The procedure is simple and basically consists of showing that a general result of Gilbert on transplantation remains true when one considers weights in the Muchkenhoupt $A_{p}$-classes.

Second we note that a lower bound on the $L^{p}$-norm of the polynomials is only needed in order to obtain a result on divergence a.e. In this case, a powerful result established by Máté, Nevai, and Totik [9], which is valid for a very general class of polynomials on $[-1,1]$, allows us to prove a general divergence theorem that includes the Badkov and Meaney results. Moreover, we are able to prove an analogous result to Máté, Nevai, and Totik's in the context of Bessel functions, which allows us to obtain divergence results in this case.

We shall use the following notations. Let $\alpha, \beta>-1$. We shall denote by $P_{n}^{(\alpha, \beta)}$ the Jacobi polynomials on $[-1,1]$ with respect to the measure $d \mu^{(\alpha, \beta)}=$ $(1-x)^{\alpha}(1+x)^{\beta} d x$ (see [15]). Let $J_{\alpha}$ be the Bessel function of order $\alpha>-1$ and $j_{n}^{\alpha}(x)=\sqrt{2}\left|J_{\alpha+1}\left(\alpha_{n}\right)\right|^{-1} J_{\alpha}\left(\alpha_{n} x\right)$ (where $\alpha_{n}$ is the increasing sequence of the zeroes of $J_{\alpha}$ ) the Bessel system of order $\alpha$, which is orthonormal and complete in $L^{2}([0,1] ; x d x)$.

The corresponding maximal operators will be denoted by $S_{\alpha, \beta}^{*}$ and $S_{\alpha}^{*}$, respectively.

$A_{p}([a, b])$ (where $\left.1<p<\infty\right)$ will stand for the Muckenhoupt classes (see [11]) consisting of those nonnegative functions $\omega$ on $[a, b]$ such that

$$
\left(|I|^{-1} \int_{I} \omega\right)\left(|I|^{-1} \int_{I} \omega^{-p^{\prime} / p}\right)^{p / p^{\prime}} \leq C \quad \text { for every interval } I \subseteq[a, b],
$$

where $p+p^{\prime}=p p^{\prime}$.

\section{Extension to $A_{p}$ Classes of a Gilbert result}

Let $\left(u_{n}\right)_{n \geq 0}$ a sequence of functions on $[0, \pi]$ verifying:

(1.1) There exists a constant $A$ such that $\sup _{0<x<\pi}\left|u_{n}(x)\right| \leq A \quad \forall n$.

(1.2) There exist functions $X_{1}, X_{2}, X_{3}, X_{4}$ in $L^{\infty}((0, \pi / 2))$ such that

$$
\begin{aligned}
u_{n}(x)= & X_{1}(x) \cos n x+X_{2}(x) \sin n x \\
& +(n x)^{-1}\left(X_{3}(x) \cos n x+X_{4}(x) \sin n x\right)+O\left((n x)^{-2}\right)
\end{aligned}
$$


uniformly for $x \in(1 / n, \pi / 2)$.

(1.3) There exist functions $X_{1}^{\prime}, X_{2}^{\prime}, X_{3}^{\prime}, X_{4}^{\prime}$ in $L^{\infty}((0, \pi / 2))$ such that

$$
\begin{aligned}
\Delta u_{n}(x)= & x\left(X_{1}^{\prime}(x) \cos n x+X_{2}^{\prime}(x) \sin n x\right) \\
& +n^{-1}\left(X_{3}^{\prime}(x) \cos n x+X_{4}^{\prime}(x) \sin n x\right)+O\left(n^{-2} x^{-1}\right)
\end{aligned}
$$

uniformly for $x \in(1 / n, \pi / 2)$, where $\Delta u_{n}=u_{n}-u_{n+1}$.

(1.4) There exists a function $X$ in $L^{\infty}((0, \pi / 2))$ such that

$$
\Delta u_{n}(x)=n^{-1} X(x) u_{n}(x)+\left(n^{-2}+x\right) O(1)
$$

uniformly for $x \in(0,1 / n)$.

(1.5) There exists a sequence $\left(U_{n}\right)$ satisfying (1.1)-(1.4) such that

$$
u_{n}(\pi-x)=(-1)^{n} U_{n}(x)+O\left(n^{-2}\right)
$$

uniformly for $x \in(0, \pi / 2)$.

Let $\left(r_{n, k}\right)$ be a double sequence such that

(1.6) $\sum_{n=0}^{\infty}\left|r_{n, k}\right|<\infty \quad \forall k$.

(1.7) There exists a constant $B$ such that $\left|r_{n, k}\right| \leq B \quad \forall n, k$.

(1.8) There exists a constant $B$ such that $\sum_{n=1}^{\infty}\left|\Delta\left(r_{n, k}\right)\right|<B \quad \forall k$, where $\Delta\left(r_{n, k}\right)=r_{n+1, k}-r_{n, k}$.

If $\left(u_{n}\right),\left(v_{n}\right)$ satisfy $(1.1)-(1.5)$ and $\left(r_{n, k}\right)$ verifies $(1.6)-(1.8)$ then we define the kernels:

$$
K_{k}(x, t)=\sum_{n=0}^{\infty} r_{n, k} e^{i n x} e^{-i n t}, \quad L_{k}(x, t)=\sum_{n=0}^{\infty} r_{n, k} u_{n}(x) v_{n}(t) .
$$

In this situation the Gilbert result is (see [5]):

Theorem. Let $1<p<\infty$. If the operator $f \rightarrow \sup _{k}\left|\int_{-\pi}^{\pi} f(t) K_{k}(x, t) d t\right|$ is of weak type $(p, p)$ or strong type $(p, p)$ in $L^{p}((-\pi, \pi))$, then the same is true for the operator $\phi \rightarrow \sup _{k}\left|\int_{-\pi}^{\pi} \phi(t) L_{k}(x, t) d t\right|$ in $L^{p}((0, \pi))$.

Examples of $\left(u_{n}\right)$ satisfying (1.1)-(1.5) are (see [5])

$$
u_{n}^{\alpha, \beta}(\theta)=A_{n}^{\alpha, \beta} P_{n}^{\alpha, \beta}(\cos \theta)(1-\cos \theta)^{\alpha / 2+1 / 4}(1+\cos \theta)^{\beta / 2+1 / 4}, \quad \alpha, \beta \geq-\frac{1}{2}
$$

(where $A_{n}^{\alpha, \beta}$ is a normalization constant), or

$$
u_{n}^{\alpha}(\theta)=\pi^{-1} \theta^{1 / 2} j_{n}^{\alpha}\left(\pi^{-1} \theta\right), \quad \alpha \geq-\frac{1}{2} .
$$

If we call $T_{\alpha, \beta}^{*}$ and $T_{\alpha}^{*}$ to the corresponding maximal operators, then we have the relations

$$
S_{\alpha, \beta}^{*} f(\cos \theta)=T_{\alpha, \beta}^{*} g(\theta)(1-\cos \theta)^{-\alpha / 2-1 / 4}(1+\cos \theta)^{-\beta / 2-1 / 4}
$$

where $g(\theta)=f(\cos \theta)(1-\cos \theta)^{\alpha / 2+1 / 4}(1+\cos \theta)^{\beta / 2+1 / 4}$ and

$$
S_{\alpha}^{*} f\left(\pi^{-1} \theta\right)=T_{\alpha}^{*} g(\theta) \theta^{-1 / 2}
$$

where $g(\theta)=f\left(\pi^{-1} \theta\right) \theta^{-1 / 2}$.

It is clear from Gilbert's theorem that it is possible to obtain boundedness for the maximal operators $T_{\alpha, \beta}^{*}$ and $T_{\alpha}^{*}$ by comparing them with the usual Carleson-Hunt maximal operator (see $[4,6]$ ) associated to the trigonometric system. However, formulae (1.9) and (1.10) indicate that we shall need a "weighted" Gilbert theorem if we want to obtain some boundedness for the maximal operators $S_{\alpha, \beta}^{*}$ and $S_{\alpha}^{*}$. 
Theorem 1. Let $1<p<\infty$. Let $\omega \in A_{p}((-\pi, \pi))$ an even weight. Let $\left(u_{n}\right),\left(v_{n}\right)$, and $\left(r_{n, k}\right)$ be sequences under the hypothesis of Gilbert's theorem. If the operator $f \rightarrow A^{*} f=\sup _{k}\left|\int_{-\pi}^{\pi} f(t) K_{k}(x, t) d t\right|$ is of weak or strong type $(p, p)$ in $L^{p}((-\pi, \pi), \omega)$, then the same is true for the operator $\phi \rightarrow B^{*} f=$ $\sup _{k}\left|\int_{-\pi}^{\pi} \phi(t) L_{k}(x, t) d t\right|$ in $L^{p}((0, \pi), \omega)$.

Proof. The Gilbert proof is based on the control of the operator $B^{*}$ by operators close to the operator $A^{*}$ (since $\left(u_{n}\right),\left(v_{n}\right)$ may be expressed in terms of sines and cosines) together with another one that can be essentially controlled by the Hardy-Littlewood maximal function. Now, the proof consists of observing that all the operators taking part in the proof are bounded with $A_{p}$ weights because of the hypothesis and Muckenhoupt's result related to the boundedness of the Hardy-Littlewood maximal function with $A_{p}$ weights (see [11]). Details can be found in [13, Chapter IV].

When $r_{n, k}=1$ if $n \leq k$ and 0 otherwise and $\left(u_{n}\right)=\left(v_{n}\right)$ is the sequence $u_{n}^{\alpha, \beta}\left(\right.$ or $\left.u_{n}^{\alpha}\right)$, then $A^{*}$ is the operator

$$
f \rightarrow S^{*}\left(f^{b}\right)(x)=\sup _{k}\left|\sum_{n=0}^{k} \hat{f}(n) e^{i n x}\right|,
$$

where $S^{*}$ is the Carleson maximal operator and $f^{b}=\hat{f}(0)+\frac{1}{2}(f+\tilde{f}), \tilde{f}$ being the conjugate function of $f$.

Both operators $S^{*}$ and $f^{b}$ are bounded in $L^{p}((-\pi, \pi), \omega)$ when $\omega$ is an $A_{p}$ weight, due to Hunt, Muckenhoupt, and Wheeden's theorem (see [7]) and Hunt and Young's theorem (see [8]). On the other hand, the corresponding operator $B^{*}$ is the maximal operator $T_{\alpha, \beta}^{*}\left(\right.$ or $\left.T_{\alpha}^{*}\right)$.

Some applications of Theorem 1 are

Theorem 2. (i) Let $\alpha, \beta \geq-\frac{1}{2}$ and $1<p<\infty$. Let $u$ be a weight on $[-1,1]$. If

$$
\omega(t)=u(\cos t)(1-\cos t)^{(2-p)(2 \alpha+1) / 4}(1+\cos t)^{(2-p)(2 \beta+1) / 4} \in A_{p}((0, \pi))
$$

then $S_{\alpha, \beta}^{*}$ is bounded in $L^{p}\left(u d \mu^{(\alpha, \beta)}\right)$.

(ii) Let $\alpha \geq-\frac{1}{2}$ and $1<p<\infty$. Let $u$ be a weight on [0,1]. If $u(x) x^{1-p / 2} \in A_{p}((0,1))$ then $S_{\alpha}^{*}$ is bounded in $L^{p}(u(x) x d x)$.

Proof. (i) By (1.9), $S_{\alpha, \beta}^{*}$ is bounded on $L^{p}\left(u d \mu^{(\alpha, \beta)}\right)$ if and only if $T_{\alpha, \beta}^{*}$ is bounded on $L^{p}(\omega)$ and this follows from the above mentioned remarks.

(ii) It is analogous using (1.10) instead of (1.9).

A particular case of Theorem 2(i) is

Corollary 1 (Badkov [2]). Let $\alpha, \beta \geq-\frac{1}{2}, \quad 1<p<\infty$, and $u(x)=$ $(1-x)^{a p}(1+x)^{b p}$. If the conditions

$$
\begin{array}{ll}
a+(\alpha+1)\left(\frac{1}{p}-\frac{1}{2}\right)<\frac{1}{4}, & b+(\beta+1)\left(\frac{1}{p}-\frac{1}{2}\right)<\frac{1}{4}, \\
a+(\alpha+1)\left(\frac{1}{p}-\frac{1}{2}\right)>-\frac{1}{4}, & b+(\beta+1)\left(\frac{1}{p}-\frac{1}{2}\right)>-\frac{1}{4}
\end{array}
$$

are fullfilled, then $S_{\alpha, \beta}^{*}$ is bounded on $L^{p}\left(u d \mu^{(\alpha, \beta)}\right)$. 
Proof. We need only to check that

$$
(1-\cos t)^{a p+\{(2-p)(2 \alpha+1)\} / 4}(1+\cos t)^{b p+\{(2-p)(2 \beta+1)\} / 4} \in A_{p}((0, \pi)),
$$

but this is equivalent to

$$
t^{2 a p+\{(2-p)(2 \alpha+1)\} / 2}(\pi-t)^{2 b p+\{(2-p)(2 \beta+1)\} / 2} \in A_{p}((0, \pi)),
$$

and a simple exercise of integration shows that this is true taking into account the hypothesis.

Note. Considering different systems $\left(u_{n}\right),\left(v_{n}\right)$, Theorem 1 also allows us to obtain transplantation's results of the following type:

Let $d \mu, d \nu$ be two different measures of Jacobi type and let $P_{n}, Q_{n}$ the corresponding orthonormal Jacobi polynomials. Then, for a certain range of $p$ 's, for each $f \in L^{p}(d \nu)$, there exists a $g \in L^{p}(d \mu)$ such that

$$
\int_{-1}^{1} g(x) P_{n}(x) d \mu(x)=\int_{-1}^{1} f(x) Q_{n}(x) d \nu(x) \quad \forall n \geq 0
$$

and $\|g\|_{L^{p}(d \mu)} \leq C\|f\|_{L^{p(d \nu)}}$ where the constant $C$ is independent of $f$.

Theorems of this type have been studied by Askey [1] and Muckenhoupt [12], not for the polynomials on $[-1,1]$ but for the corresponding orthonormal functions in $(0, \pi)$.

\section{DIVERGENCE ALMOST EVERYWHERE}

First we are going to study the case of orthonormal polynomials in $[-1,1]$. Assume $d \mu$ is a positive Borel measure on $[-1,1]$ such that $\mu^{\prime}>0$ a.e., and let $\left\{P_{n}(x)\right\}_{n=0}^{\infty}$ denote the corresponding orthonormal polynomials.

The following result is due to Máté, Nevai, and Totik [9].

Lemma 1. Suppose $1 \leq r<\infty$. If $g$ is a Lebesgue measurable function in $[-1,1]$, then

$$
\left(\int_{-1}^{1}\left|g(x)\left(1-x^{2}\right)^{-1 / 4} \mu^{\prime}(x)^{-1 / 2}\right|^{r} d x\right)^{1 / r} \leq M \liminf _{n \rightarrow \infty}\left(\int_{-1}^{1}\left|g(x) P_{n}(x)\right|^{r} d x\right)^{1 / r}
$$

where $M=\pi^{1 / 2} 2^{\max \{1 / r-1 / 2,0\}}$.

By using this lemma we obtain

Theorem 3. Let $\omega$ be a Lebesgue measurable positive function in $[-1,1]$ and $1<p \leq q<\infty,(1 / p+1 / q=1)$. If

$$
\omega(x)^{-1} \notin L^{q}(d \mu) \quad \text { or } \quad \mu^{\prime}(x)^{1-q / 2}\left(1-x^{2}\right)^{-q / 4} \omega(x)^{-q} \notin L^{1}(d x),
$$

then there exists $f \in L^{p}\left(\omega^{p} d \mu\right)$ such that $S_{n} f$ either does not exist or diverges almost everywhere in $[-1,1]$.

Proof. Suppose that $c_{0}(f)=\int_{-1}^{1} f(x) P_{0}(x) d \mu(x)$ exists for each $f \in$ $L^{p}\left(\omega^{p} d \mu\right)$. By duality, this implies $P_{0} \in L^{q}\left(\omega^{-q} d \mu\right)$, i.e., $\omega^{-1} \in L^{q}(d \mu)$. 
If $\omega^{-1} \in L^{q}(d \mu)$, then $S_{n} f$ exists for every $n=0,1,2, \ldots$ and every $f \in L^{p}\left(\omega^{p} d \mu\right)$, and so the operators

$$
c_{n}: f \in L^{p}\left(\omega^{p} d \mu\right) \rightarrow c_{n}(f)=\int_{-1}^{1} f(x) P_{n}(x) d \mu(x)
$$

are continuous by using Hölder's inequality. Moreover, $\left\|c_{n}\right\|=\left\|P_{n} \omega^{-1}\right\|_{L^{q}(d \mu)}$ and so, by (2.1) and Lemma 1, it is clear that $\sup _{n}\left\|P_{n} \omega^{-1}\right\|_{L^{q}(d \mu)}=\infty$. Then the Banach-Steinhaus theorem shows that there exists $f \in L^{p}\left(\omega^{p} d \mu\right)$ such that $\sup _{n}\left|c_{n}(f)\right|=\infty$.

On the other hand, if $S_{n} f(x)=\sum_{k=0}^{n} c_{k}(f) P_{k}(x)$ converges in a set $E$ of Lebesgue measure $|E|>0$ and $\sup _{n}\left|c_{n}(f)\right|=\infty$, then there exists a subsequence $P_{n_{k}}$ with

$$
\lim _{k} P_{n_{k}}(x)=0 \quad \text { a.e. in } E \text {. }
$$

If we take $\delta>0$ and $\delta<|E|$, by using Egoroff's theorem, there exists $D \subset E$ such that $|D|=\delta$ and $\lim _{k} P_{n_{k}}(x)=0$ uniformly in $D$. Therefore

$$
\lim _{k} \int_{D}\left|P_{n_{k}}\right| d \mu=0 \text {. }
$$

But, by Lemma 1 with $r=1$ and $g(x)=\mu^{\prime}(x) \chi_{D}(x)$, we have

$$
\lim _{k} \int_{D}\left|P_{n_{k}}\right| d \mu \geq M^{-1} \int_{D}\left(1-x^{2}\right)^{-1 / 4} \mu^{\prime}(x)^{1 / 2} d x>0,
$$

which is a contradiction.

Next we show a similar result for Bessel systems. First of all, we need to establish an analogous result of Lemma 1. For that, we shall use the following estimates for Bessel functions

$$
\begin{gathered}
J_{\alpha}(z)=z^{\alpha} 2^{-\alpha} \Gamma(\alpha+1)^{-1}+O\left(z^{\alpha+2}\right), \quad z \rightarrow 0, \\
J_{\alpha}(z)=\sqrt{2}(\pi z)^{-1 / 2} \cos \left(z-\frac{\alpha \pi}{2}-\frac{\pi}{4}\right)+O\left(z^{-3 / 2}\right), \quad z \rightarrow \infty, \\
\int_{0}^{1} J_{\alpha}\left(\alpha_{n} x\right)^{2} x d x \approx \frac{1}{\pi \alpha_{n}}, \quad n \rightarrow \infty
\end{gathered}
$$

and the Fejer lemma: if $f, g$ are nonnegative functions, $g$ is continuous with period $2 \pi$, and $f \in L^{1}((0,2 \pi))$, then

$$
\lim _{\lambda \rightarrow \infty} \frac{1}{2 \pi} \int_{0}^{2 \pi} f(t) g(\lambda t) d t=\hat{g}(0) \hat{f}(0)
$$

where $\hat{h}(x)$ denotes the Fourier transform of $h$.

Lemma 2. Let $\alpha>-1$. Let $h(x)$ be a Lebesgue measurable nonnegative function on $[-1,1]$ and $1<p<\infty$. Then

$$
\lim _{n \rightarrow \infty} \int_{0}^{1}\left|j_{n}^{\alpha}(x)\right|^{p} h(x) d x \geq M \int_{0}^{1} h(x) x^{-p / 2} d x
$$

where

$$
M=\frac{2^{p / 2}}{2 \pi} \int_{0}^{2 \pi}|\cos t|^{p} d t .
$$


Proof. If there exists no $\delta \in(0,1)$ such that $h(x) x^{\alpha p} \notin L^{1}(0, \delta)$ then (2.6) is trivial since (2.2) implies that all the integrals on the left in (2.6) are equal to $\infty$. Then, we may suppose that $h(x) x^{\alpha p} \in L^{1}(0, \delta)$ for some $\delta \in(0,1)$. By (2.4) it is enough to prove

$$
\lim _{n \rightarrow \infty} \int_{0}^{1}\left|\left(\pi \alpha_{n}\right)^{1 / 2} J_{\alpha}\left(\alpha_{n} x\right)\right|^{p} h(x) d x=M \int_{0}^{1} h(x) x^{-p / 2} d x .
$$

Suppose that $h(x)^{-p / 2} \in L^{1}((0,1))$. If we prove

$$
\lim _{n \rightarrow \infty} \int_{0}^{1}\left|\left(\alpha_{n} x\right)^{1 / 2} J_{\alpha}\left(\alpha_{n} x\right)-\left(\frac{2}{\pi}\right)^{1 / 2} \cos \left(\alpha_{n} x-\frac{\alpha \pi}{2}-\frac{\pi}{4}\right)\right|^{p} h(x) x^{-p / 2} d x=0,
$$

then $(2.7)$ is reduced to show that

$$
\lim _{n \rightarrow \infty} \int_{0}^{1}\left|\left(\frac{2}{x}\right)^{1 / 2} \cos \left(\alpha_{n} x-\frac{\alpha \pi}{2}-\frac{\pi}{4}\right)\right|^{p} h(x) d x=M \int_{0}^{1} h(x) x^{-p / 2} d x
$$

Now (2.9) holds by using (2.5) with

$$
\begin{gathered}
t=2 \pi x, \quad g(t)=\left|2^{1 / 2} \cos \left(t-\frac{\alpha \pi}{2}-\frac{\pi}{4}\right)\right|^{p}, \\
f(t)=h\left(\frac{t}{2 \pi}\right)\left(\frac{t}{2 \pi}\right)^{-p / 2}, \quad \lambda=\frac{\alpha_{n}}{2 \pi} .
\end{gathered}
$$

Thus, let us prove (2.8) under conditions

$$
h(x) x^{\alpha p} \in L^{1}((0, \delta)) \text { and } h(x) x^{-p / 2} \in L^{1}((0,1)) .
$$

Take $n$ large enough so that $\alpha_{n}>1$. In view of (2.4), if $\alpha_{n} x \geq 1$ then $\left|\left(\alpha_{n} x\right)^{1 / 2} J_{\alpha}\left(\alpha_{n} x\right)-\left(\frac{2}{\pi}\right)^{1 / 2} \cos \left(\alpha_{n} x-\frac{\alpha \pi}{2}-\frac{\pi}{4}\right)\right| \leq C\left(\alpha_{n} x\right)^{1 / 2}\left(\alpha_{n} x\right)^{-3 / 2}<C_{1}$, and, according to (2.2), if $\alpha_{n} x \leq 1$ it follows

$$
\begin{gathered}
\left|\left(\alpha_{n} x\right)^{1 / 2} J_{\alpha}\left(\alpha_{n} x\right)-\left(\frac{2}{\pi}\right)^{1 / 2} \cos \left(\alpha_{n} x-\frac{\alpha \pi}{2}-\frac{\pi}{4}\right)\right| \\
\leq C\left(\left(\alpha_{n} x\right)^{\alpha+1 / 2}+1\right) \leq C_{1}\left(x^{\alpha+1 / 2}+1\right)
\end{gathered}
$$

(in the last inequality we use $\alpha_{n} x \leq 1$ if $\alpha+\frac{1}{2} \geq 0$ and $\alpha_{n} x \geq x$ if $\alpha+\frac{1}{2} \leq 0$ ). Now, by (2.10), we can apply the dominate convergence theorem and obtain (2.8).

Finally, in case that $h(x) x^{-p / 2} \notin L^{1}((0,1))$, let $\left\{K_{j}\right\}_{j=1}^{\infty}$ be the sequence of increasing measurable sets $K_{j}=\left\{x \in(0,1): h(x) x^{-p / 2} \leq j\right\}$ and denote by $h_{j}$ the function $h_{j}=h$ on $K_{j}$ and 0 elsewhere. By applying (2.7) for each $h_{j}$ and then the monotone convergence theorem, (2.7) also holds in this case, and the proof of the lemma is finished.

From Lemma 2 and proceeding exactly as in the proof of Theorem 3, we obtain 
Theorem 4. Let $\alpha>-1$ and suppose $1<p \leq q<\infty,(1 / p+1 / q=1)$. Let $\omega$ be a Lebesgue measurable nonnegative function on $[0,1]$. If

$$
\omega(x)^{-q} x^{\alpha q+1} \notin L^{1}(d x) \quad \text { or } \quad \omega(x)^{-q} x^{1-q / 2} \notin L^{1}(d x)
$$

then there exists $f \in L^{p}\left(\omega(x)^{p} x d x\right)$ whose Fourier series with respect to the system $\left\{j_{n}^{\alpha}(x)\right\}$ either does not exist or diverges almost everywhere on $[0,1]$.

Notes. (i) If $\alpha \geq-\frac{1}{2}$, the proof of Lemma 2 shows that the sign " $\geq$ " can be replaced by "=".

(ii) Theorems 3 and 4 contain as particular cases the known cases of generalized Jacobi polynomials [2, 10, 14] or Bessel systems [3].

(iii) Analogous result to Theorem 4 can be obtained for Dini systems in $[0,1]$.

\section{REFERENCES}

1. R. Askey, A transplantation theorem for Jacobi series, Illinois J. Math. 13 (1969), 583-590.

2. V. M. Badkov, Convergence in the mean and almost everywhere of Fourier series in polynomials orthogonal on an interval, Math. USSR Sb. 24 (1974), 223-256.

3. A. I. Benedek and R. Panzone, Pointwise convergence of series of Bessel functions, Rev. Un. Mat. Argentina 26 (1972), 167-186.

4. L. Carleson, On convergence and growth of partial sums of Fourier series, Acta Math. 116 (1966), 135-157.

5. J. E. Gilbert, Maximal theorems for some orthogonal series. I, Trans. Amer. Math. Soc. 145 (1969), 495-515.

6. R. Hunt, On the convergence of Fourier series, Proc. Conf. Orthogonal Expansions and Continuous Analogues, Southern Illinois Univ. Press, Carbondale, IL, 1968, pp. 235-255.

7. R. Hunt, B. Muckenhoupt, and R. Wheeden, Weighted norm inequalities for the conjugate function and Hilbert transform, Trans. Amer. Math. Soc. 176 (1973), 227-251.

8. R. Hunt and W. S. Young, A weighted norm inequality for Fourier series, Bull. Amer. Math. Soc. 80 (1974), 274-277.

9. A. Máté, P. Nevai, and V. Totik, Necessary conditions for weighted mean convergence of Fourier series in orthogonal polynomials, J. Approx. Theory 46 (1986), 314-322.

10. C. Meaney, Divergent Jacobi polynomial series, Proc. Amer. Math. Soc. 87 (1983), 459-462.

11. B. Muchenhoupt, Weighted norm inequalities for the Hardy maximal function, Trans. Amer. Math. Soc. 165 (1972), 207-226.

12. _ Transplantation theorems and multiplier theorems for Jacobi series, Mem. Amer. Math. Soc., vol. 64, no. 356, Amer. Math. Soc., Providence, RI, 1986.

13. M. Pérez, Series de Fourier respecto de sistemas ortogonales: estudio de la convergencia en espacios de Lebesgue y de Lorentz, Doctoral Dissertation, Sem. Mat. García de Galdeano, Zaragoza, 1989.

14. H. Pollard, The convergence almost everywhere of Legendre series, Proc. Amer. Math. Soc. 35 (1972), 442-444.

15. G. Szegö, Orthogonal polynomials, 3rd ed., Amer. Math. Soc., Providence, RI, 1967.

16. E. C. Titchmarsh, Eigenfunction expansions associated with second-order differential equations, Vol. 1, 2nd ed., Oxford Univ. Press, Oxford, 1962.

(José J. Guadalupe, Mario Pérez and Francisco J. Ruiz) Departamento de Matemáticas, Facultad de Ciencias, Universidad de Zaragoza, 50009 Zaragoza, Spain

(Juan L. Varona) Departamento de Matemática Aplicada, Colegio Universitario de La RIOJA, 26001 Logroño, SPAIN

E-mail address: JVARONA@CC.UNIZAR.ES 